\title{
Exploring the Potential Use of Adhesion Strength for Coating Analysis
}

\author{
Yunan Prawoto, Nazri Kamsah, and Zaini Ahmad
}

\begin{abstract}
This paper discusses the possibility of the potential use of adhesion strength for coating analysis. Most of the steel structures used in industrial and non-industrial applications are exposed to outdoors weathering conditions. Organic coating typically protects them from corrosion. The maintenance actions can be done efficiently only if there is sufficient information on the accurate condition of it. Therefore, the deterioration of the coating system and its lifetime has to be assessed accurately. Adhesion strength has the potential to be used as a parameter for evaluation. This paper explores the development of these parameters mainly based on fracture mechanics.
\end{abstract}

Index Terms-Blister, coating degradation, fracture mechanics, organic coating.

\section{INTRODUCTION}

The cost to repair coating failures can easily overshadow the initial cost of painting. This leads to significant amount of financial loss that is incurred every year as a result of premature failures of paints and coatings. Additional liability may also be expected if the operation of the facility has to be stopped for repair work. This is the rationale of the coating life assessment and failure analysis. It was proposed that coating deterioration and degradation could be modeled in three ways [1]:

- As a black-box of statistical time to failure (such as lifetime distribution);

- As a grey-box of stress-strength model based on a measurable quantity indicating time-dependent deterioration and failure;

- As a white-box model through a simulation of the physics of measurable deterioration and failure.

In this research, the focus is given on the second and the third methods with some manipulation similar to that of life assessment methodology for metal structures [2]- [4]. A number of papers have been published on the degradation of systems exposed to outdoor weathering conditions. For example, Chan and Meeker [5] relate degradation to environmental factors, such as the weather. These factors are

Manuscript received on March 4, 2012. Funding is by the MOHE (Ministry of Higher Education), Government of Malaysia through Research University Grant (RUG-GUP) UTM number Q. J130000. 7124. 00H14, under the title of Degradation of corrosion protective coatings on steel: computational and experimental approaches to blistering formation and development.

Y. Prawoto is a faculty member at the Universiti Teknologi Malaysia. (yunan.prawoto@gmail.com). transformed into a degradation rate. A time series modeling approach was proposed to predict daily degradation. Heutink et al. [6] describe how the maintenance methodology used in the Netherlands was applied to protective paint systems. The lifetime-extending maintenance model, in which deterioration is modeled by a gamma process with expected deterioration non-linear in time, is applied successfully to optimize maintenance of the coating of the Haringvliet storm-surge barrier. Among other deterioration parameters, this paper emphasizes on the adhesive strength taking the advantage of the blister formation and development as a symptom of the coating deterioration.

\section{BLISTERING IN COATING}

Blisters are local defects due to the pressure exerted by an accumulation of substrate at the coating-substrate interface in conjunction with loss of adhesion and distention of the coating. At these local regions, corrosion of the substrate may occur. Typically the loss of coating adhesion is related to the development of a cathodic area under the coating adjacent to the defect. Oxygen also permeates into the coating while ionic materials are leached from the substrate or from the coating and all these effects will combine together to form an electrochemical corrosion cell beneath the blister. Therefore blisters are an early sign of corrosion but are often neglected. Conversely, the elimination, reduction, or delay in blister formation will delay the onset of corrosion of the steel substrate.

Several different forms and mechanisms for blister formation are postulated; the most likely possibilities are identified here. In general, the mechanism of blistering is attributed to osmotic attack or the presence of defects in the coating interfacial region, in combination with the influence of moisture. The following is a sequence of events leading to the formation of most types of blistering [7, 8]:

o The film absorbs water from a solution possibly containing dissolved salts. Or water and/or corrosive substance may enter through some coating damage such as defect or cut in the coating layer.

- Once sufficient amount of chloride ions pass through to the underlying metal, a primary corrosion is initiated at the sites along the interface, particularly at any existing defective areas or areas of contaminated substrate.

- As the corrosion proceeds at the anodic sites under the film, ions build up at the cathodic sites.

- The alkaline environment at the cathodic sites weakens or destroys the adhesion of the film while 
producing osmotically active substances at the coating/metal interface.

o The presence of these active substances at the interface causes osmotic (or endosmotic) passage of water from the coating surface to the interface. This results in the development of pressures that exceed the interfacial strength of the film and eventually the fracture strength of the film, causing further deadhesion or coating rupture.

Several mechanisms are generally proposed to explain blister formation phenomenon: volume expansion due to swelling, gas inclusion or gas formation, electroendosmotic blistering, osmotic blistering, and cathodic blistering.

The idea of coating degradation is similar to that of the delamination [9] that metallurgical failure analysts are familiar with. Fig. 1 shows how a typical delamination is found in the field.

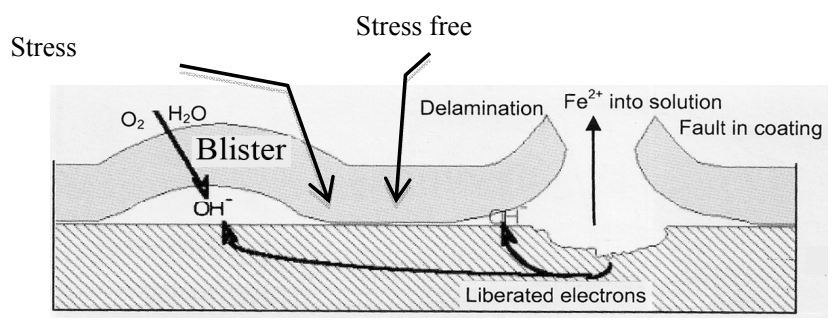

Fig. 1. Corrosion delamination mechanism [9].

In the metal/composite society, the delamination is often approached by using the fracture mechanics concepts. In this research, mimicking those approaches, fracture mechanics approach is used.

A large variety of outdoor and laboratory equipment and procedures have been used for decades [10] - [12]. Outdoor exposures are reliable and offer a good representation of the actual service life. The data collected will provide a basis for the selection of particular coatings for specific applications and they also provide an insight into how new coatings could be formulated. However, outdoor exposure is slow. Even with outdoor exposure sites being located at places where solar insulation and/or humidity are maximized, the acceleration factor is only of the order of twice, compared to more temperate climes. Greater acceleration can be achieved outdoors by concentration of the sun's rays, or indoors by a variety of methods, such as salt-spray testing. Standardized methods (e.g. ASTM) for accelerated testing are available [12]. As early as the 1990's, the prohesion test, cycling between salt immersion, salt fog, and dry off, were supplemented with alternate UV in the standard [12]. An alternate cycle of UV and continuous condensation was proposed in 1991 [13], [14]. Variation of the ASTM cycle and additional low temperature stress was also proposed several years later [15]. In the automotive industries, the most famous coating assessment is part of Ford Key Life test. It is a system or sub-system based test designed to demonstrate failure modes within the expected useful life e.g., 10 years/150,000 miles. The testing is based on product specific data and correlates closely to the actual range of usage conditions, where a certain cycle of testing corresponds to a certain years of usage. The coating evaluation basically combines the following: salt fog, humidity fogging, dry cycle, solution spray, and immersion cycle. Despite being accelerated, these tests usually require thousands of hours to be completed before damage evaluation can be done. On top of that, the damage evaluation is almost always subjective; depends on the ability of the operator. In addition, it remains that the greater the acceleration factor, the less reliable the result, both in terms of predicting end of life in real terms, and even the mode of failure [16].

\section{REVIEW OF RELATED WORKS}

The first model to use the mechanics concept was developed as early as 1995 [17]. Since then, this method has become increasingly popular in this area of research e.g., $[18,19]$. This concept was formulated by using the energy balance principle. The total energy $U_{T}$ of a blistered body can be written as:

$$
U_{T}=U_{P}+U_{E}+U_{S}
$$

where $U_{P}$ is potential energy of externally applied load, $U_{E}$ is elastic energy stored in the system, and $U_{S}$ is surface energy. If the fresh area created by blister is $d A$, three different cases can be thought, namely

- Case 1 , Crack retreat, $d A$ is negative.

- Case 2, Equilibrium, No crack propagation, $d A$ is zero, and

○ Case 3, Crack propagate, $d A$ is positive.

The potential energy is given by:

$$
U_{P}=-\left.\int p d V\right|_{p}=-p V=-C_{1} \pi a^{2} y_{c} p
$$

where $p$ is net pressure, $a$ is blister radius, and $y_{c}$ is the deflection (or blister height).

The elastic energy can be expressed as,

$$
U_{E}=-\left.\int p\left(y_{c}\right) d V\right|_{p}=\frac{p V}{4}=\frac{C_{1} \pi a^{2} y_{c} p}{4}
$$

Assuming that the above condition resembles those of plain stress and simplified into mode I problem, the stress intensity factor can be written as:

$$
K_{I}=\sqrt{\frac{5 C_{1}}{4} E \cdot y_{c} \cdot p}
$$

where the value of $C_{1}$ is fitted with the experimental results.

Some researchers used energy method based on the fracture mechanics parameters namely effective normal $(N)$ and shear $(S)$ membrane stresses and the bending moment $(\mathrm{M})$ [20]. Expressed in SIF, Eqn. (4) becomes,

$$
K_{I}=\sqrt{\frac{6\left(1-v^{2}\right)}{h^{3}}\left(M^{2}+\frac{h^{2} N^{2}}{12}\right)+\frac{S^{2}\left(1+v^{2}\right)}{h}}
$$


where $v$ is the Poisson's ratio of the coating layer, and $h$ is the coating thickness. Eqn. (5) can be used with extensive knowledge of the coating conditions, including normal $(N)$ and shear $(S)$ membrane stresses and the bending moment $(M)$.

\section{Fracture MeChanics APPROACH IN Blistering DEVELOPMENT/ PROPAGATION}

The fracture mechanics approach is capable of handling failures involving almost any types of crack propagations. Regardless of the types of the blistering, it is evident that it can be used as a parameter to estimate the lifetime of the coating. This is very convenient since it is strongly related to adhesion strength. However, to use the adhesion strength directly would be impossible, since its value is dependent on so many factors. Therefore, three approaches based on fracture mechanics are postulated. Fracture mechanics deals with the study of the propagation of cracks in materials. Its applications on areas other than mechanics are many, especially in materials science [20].

In principle, our selection of the parameter must be able to describe and accommodate the stress singularity at the crack tip, as shown in Fig 1. Near the tip, where the blister is propagating, is defined as stress singularity area. The pressure difference between the atmospheric pressure and internal pressure of the blister is the driving force for the crack propagation, which in this case is blister propagation.

\section{A. Using Stress Intensity Factor (SIF):}

SIF is typically used to govern crack propagation in a brittle material. The SIF due to the pressure difference $p$ can be converted to SIF analytically or computationally. The use of the weight function is one of the easiest ways to derive the SIF. The detailed discussion on the method is available elsewhere [20, 21]. Mimicking the concept used by pioneers of fracture mechanicians, Rice and Bueckner, the weight function $h(x, r)$ is introduced here [20]- [23]:

$$
K_{2}=\int_{0}^{r} \sigma(x) \cdot h(x, r) d x
$$

The displacement function $u(x, r)$, in this case was obtained by experiment. The polynomial curve fitting in this case is:

$$
u(x, r)=y_{c} \cdot \sum_{i=0}^{n}\left(\frac{x}{r}\right)^{i}
$$

where $y_{c}$ is the height of the blister and $r$ is the blister radius, and $x$ is the distance from the center of the blister. For plane strain condition, the relation between reference stress intensity factor $\left(K_{\mathrm{r}}\right)$ and the displacement function becomes:

$$
K_{r}^{2}=H \int_{0}^{r} \frac{\partial u(x, r)}{\partial r} d x
$$

Therefore, the weight function becomes:

$$
h(x, r)=\frac{H}{\sqrt{\alpha \cdot y_{c}}} \int_{0}^{r} \frac{\partial u(x, r)}{\partial r}
$$

For the case where the internal and external pressure difference is $p$, the stress part is the one responsible for mode $\mathrm{I}$; vertical component of the $p$, which is $p_{\mathrm{y}}$, taking into consideration of the integration boundary and the path, the mode I SIF becomes:

$$
K_{I}=\int_{0}^{r} p_{y} \cdot\left(\frac{E /\left(1-v^{2}\right)}{\sqrt{\alpha \cdot y_{c}}}\right) \frac{\partial u(x, r)}{\partial r} d x
$$

In here the SIF of modes I and II respectively become:

$$
\begin{gathered}
K_{I}=\alpha \cdot p_{y} E \sqrt{y_{c}} /\left(1-v^{2}\right) \\
K_{I I}=\alpha \cdot p_{x} E \sqrt{y_{c}} /\left(1-v^{2}\right)
\end{gathered}
$$

where $K_{\mathrm{I}}$ and $K_{\mathrm{II}}$ are the mode I and mode II of stress intensity factors, $p$ is the pressure difference (adhesion strength), $E$ is the Poisson's ratio and $\alpha$ is the blister shape function and $y_{c}$ is the blister height.

Therefore, based on the SIF, the $K$ parameter becomes:

$$
K=\alpha \cdot p E \sqrt{y_{c}} /\left(1-v^{2}\right)
$$

\section{B. Using Strain Energy Density (SED):}

Based on SED a mixed mode analysis can be carried out a lot easier. We have published the result of the parameter using SED elsewhere [21]. Our proposed parameter using SED is as follows:

$$
S E D=\frac{\alpha \cdot E \sqrt{y_{c}}}{\left(1-v^{2}\right)}\left(a_{11} \cdot p^{2}{ }_{x}+2 a_{12} \cdot p_{x} \cdot p_{y}+a_{22} \cdot p_{y}^{2}\right)
$$

where SED is the strain energy density factor, $p$ is the pressure difference (adhesion strength), $E$ is the Poisson' $\mathrm{s}$ ratio and $\alpha$ is the blister shape function.

\section{Using J-Integral (J-int):}

$\mathrm{J}$-integral is a way to calculate the strain energy release rate, or work (energy) per unit fracture surface area. The J-integral is equal to the strain energy release rate for a crack in a body subjected to loading under quasi-static, see Fig 3. Simplified two dimensional equation of it is:

$$
J \cong \int_{\Gamma}\left\lfloor W d x_{2}-t \frac{\partial u}{\partial x_{1}} d s\right\rfloor
$$

where $x_{1}$ is the crack direction, and $x_{2}$ is perpendicular to the crack direction, and $W$ is the strain energy density, and $n$ is the surface traction vector. The general expression is known 
as:

$$
J \cong \int_{\Gamma}\left\lfloor W n_{i}-n_{j} \sigma_{j k} \frac{\partial u_{k}}{\partial x_{1}}\right\rfloor d \Gamma
$$

where

$$
T=n_{j} \sigma_{j k}
$$

and

$$
W=\int_{0}^{\varepsilon_{i j}} \sigma_{i j} d \varepsilon_{i j}
$$

This represents the strain energy release rate of non-linear elastic materials, as shown in the following:

$$
J=\frac{d \Pi}{d A}
$$

Fig. 2 Application of J-integral in coating delamination.

Therefore, J-integral is the extension of SIF in term of the stress linearity coverage. While the SIF is popular in governing the crack propagation in brittle material, J-integral is also widely accepted in non-linear elastic material. The drawback of this method is its difficulty in the usage analytically outside the area of linear elastic. Within the area of linear elastic, its value is strongly related to SIF. However, outside the linear elastic area, practically it relies upon computational approach alone. Fig. 3 shows how to compute the J-integral with commercial finite element packages.

\section{HOW THE PARAMETERS ARE USED}

The parameters can be used for both coating life assessment (fitness for service) and failure analysis. For both cases, it is required that the relationship such as depicted in Fig 4 is determined, usually in the laboratory. The time starts with $t_{0}$, where the coating is applied, and ends at tend, where the coating has chemically deteriorated, while $t_{\mathrm{C}}$ is the time where coating is no longer usable.

The diagram is best created in the laboratory. The advantage of this approach is that any accelerated test module can be used, because the time shown in the figure is similar to that of normalized time, mimicking what is usually done in metal life assessment due to creep failure. That is, it is not necessary to know that $t_{\mathrm{b}}$ represents $x$ years, but rather that it corresponds to $70 \%$ of the effective coating life span. Once the basic data is obtained, whether by outdoor or indoor accelerated test, the diagram can then be used very easily and conveniently. The basic characterization involves obtaining the data of critical parameter, which can be easily computed from the adhesion test. How this approach is used for life assessment can be illustrated as follows: For major projects, say a steel jetty extending kilometer offshore, where one or two coatings are used, the parameter curve is determined at the outside, with deterioration accelerated in some way.

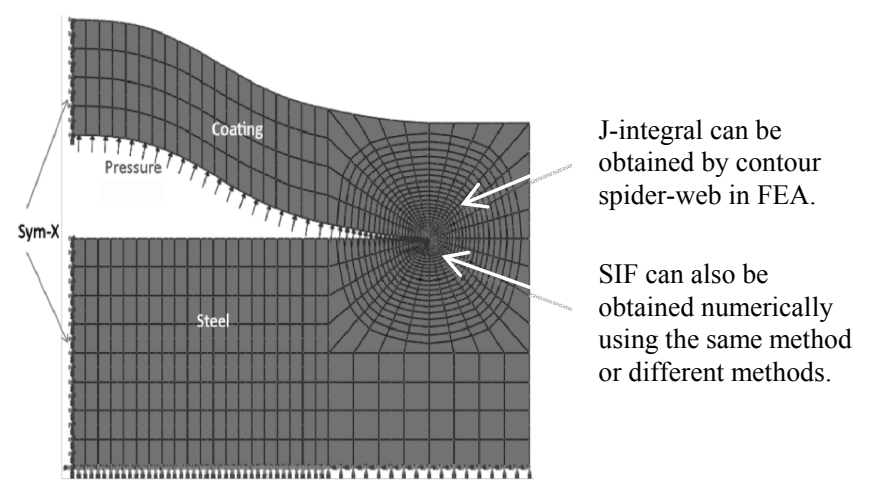

Fig. 3. Modeling of the coating blistering using FEA to obtain stress singularity parameters, such as SIF, SED, or J-int.

This curve is then available for coating evaluation at any time in the coating life. The determination of the curve at the outset, years from when it might be needed, might seem an unnecessarily complication, but is no different from the current practice with steel structures like tanks or pipelines, where thickness testing is performed at the outset to provide a base level for future comparison. It is rare for all of a coating that is used over a large project to deteriorate at the same rate.

For example it may be found that the coating on the roof and one side of a tank is close to the end of its life, because it is exposed to direct sunlight, whereas the coating on the unexposed side is only $50 \%$ spent. How this approach helps failure analysis is illustrated as follows: Similar to the computational approach of stress analysis being used in failure analysis of metals, the parameter curve can improve the failure analysis of coatings significantly. It changes a qualitative report into a quantitative one. For failure analysis to be required, some portion of the coating will already be judged to have failed. If by chance the parameter curve exists, then by field measurement of adhesion at various locations, 
one can determine if the small area of failure is representative of the coating over the structure as a whole, or whether it is atypical and most of the coating has many years of life left. Say, area $X$ is visually and mechanically in good condition. A failure analyst needs to measure the adhesion of that particular area. Subsequently, he needs to compute its critical parameter value. This value can be used to replace critical value at $t_{0}$. Area $Y$ is the one that is failed; among the visibly failed area, the adhesion test is then performed. Subsequently, the critical parameter on that area is obtained. This will be comparable to that of at $t_{\text {end }}$. The failure analyst can then sample the adhesion measurement at various locations representing different levels of performance, and combine the data to generate a map of the entire structure and present it to the customer. If the root cause is found, let's say due to the presence of particular chemical species at a certain concentration at area $Y$, then the customer can take definite yet possibly less drastic and less costly action since the failure analyst can show that the same chemical with lower concentration on area $Z$ is not detrimental enough to reduce the critical parameter.

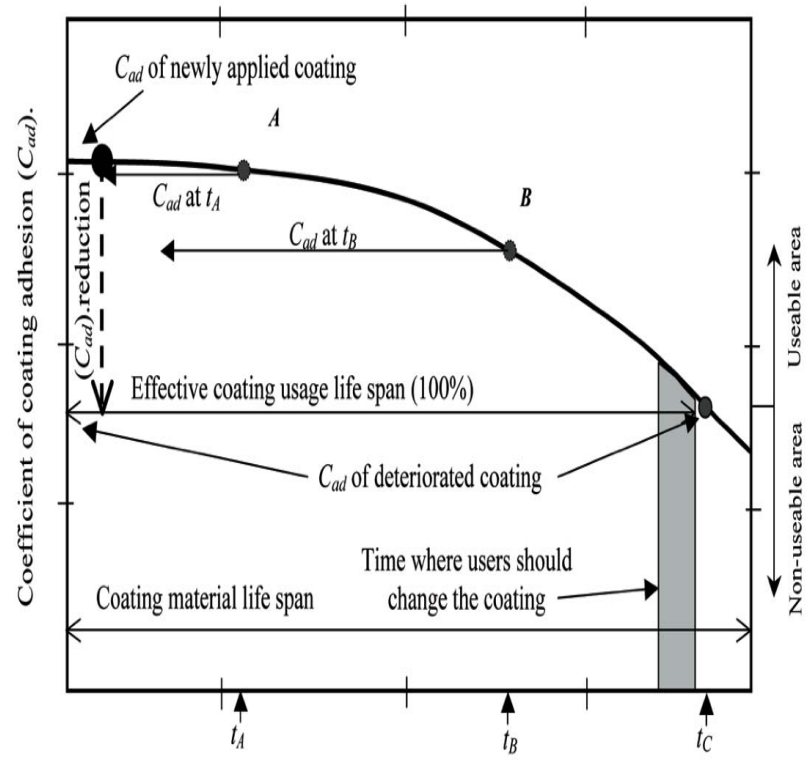

Time

Fig. 4. Example of the parameter usage.

Three conditions are postulated:

- Case 1: Crack retreat, in which parameter computed is less than critical parameter.

- Case 2: Equilibrium, in which parameter is equal to the critical parameter, and

- Case 3: Crack propagates, in which parameter is greater than critical parameter.

The critical parameter is similar to that of a metal, can be used to characterize and determine for either a new sample or a corroded sample that its adhesion is already degrading. It is a dynamic factor that changes throughout the life of the coating. The absolute time is no longer important. What is important is the normalized time with respect to the total life.

\section{CONCLUSION}

Fracture mechanics methods are used to explore new parameters for coating (degradation) life assessment. Three approaches are considered; stress intensity factor, strain energy density, and J-integral. From the practical point of view, among the three approaches, the stress intensity factor is the easiest one to use. But it also carries the drawback of being least reliable. The J-integral approach, on the other hand, is the most accurate method. However, it is not practical since it involves complex computation and modeling using the finite element method. It is worth noting that this paper is written at the early stage of the research and therefore supporting experimental data is not yet available.

\section{REFERENCES}

[1] R. P. Nicolai, R. Dekker, and J. M. van Noortwijk, "A comparison of models for measurable deterioration: An application to coatings on steel structures," Reliability Engineering \& System Safety, vol. 92, no.12, pp. 1635-1650, 2007.

[2] A. Andrae and O. Andersen, "Life cycle assessment of integrated circuit packaging technologies," The International Journal of Life Cycle Assessment, pp. 1-10, 2011.

[3] P. Puri, P. Compston, and V. Pantano, "Life cycle assessment of australian automotive door skins," The International Journal of Life Cycle Assessment, 2010.

[4] T. H. Lee and U. C. Lee, "Safeguard assessment for life extension in NPPS using a production function," Nuclear Engineering and Design, no. 241, pp.826-831, 2011.

[5] V. Chan and W. Q. Meeker, "Estimation of degradation-based reliability in outdoor environments," Technical report, Iowa State University, Ames, 2001.

[6] A. Heutink, A. van Beek, J. M. van Noortwijk, H. E. Klatter, and A. Barendregt, "Environment-friendly maintenance of protective paint systems at lowest costs," In: Proc. of 27th FATIPEC, 19-21 April 2004, France. AFTPVA; pp. 351-364, 2004.

[7] J. Pommersheim, T. Nguyen, Z. Zhang, and J. B. Hubbard, "Degradation of organic coatings on steel," Prog. in Organic Coatings, vol. 25, pp. 23-41, 1994.

[8] T. Nguyen, J. B. Hubbard, and G. B. McFadden, "Mathematical model for the cathodic blistering of organic coatings on steel immersed in electrolytes," J. of Protective Coating Tech., vol. 63, no. 794, pp. 43-52, 1991.

[9] M. Dogan, "Delamination failure of steel single angle sections," Engineering Failure Analysis, vol. 18, pp. 1800-1807, 2011.

[10] ASTM. Standard practice for cyclic salt fog/uv exposure of painted metal, (alternating exposures in a fog/dry cabinet and a uv/condensation cabinet). In ASTM: D5894. ASTM International Publisher, West Conshohocken, PA., 2009.

[11] ASTM. Standard practice for modified salt spray (fog) testing. In ASTM:G5. ASTM International Publisher, West Conshohocken, PA., 2009.

[12] ASTM. Standard test method for evaluation of painted or coated specimens subjected to corrosive environments. In ASTM:D1654. ASTM International Publisher, West Conshohocken, PA., 2008.

[13] B. S. Skerry, C. H. Simpson, and G. R. Wilson, "Combined corrosion/weathering testing of coated steel products for automotive applications," In Proceedings -Society of Automotive Engineers, pages 143-153, 1991.

[14] C. H. Simpson, C. J. Ray, and B. S. Skerry, “Accelerated corrosion testing of industrial maintenance paints using a cyclic corrosion weathering method," Journal of Protective Coatings and Linings, vol. 8 , no. 5, pp. 28-36, 1991.

[15] S. L. Chong, "Comparison of accelerated tests for steel bridge coatings in marine environments," Journal of Protective Coatings and Linings, vol. 14, no. 3, 1997.

[16] Y. Prawoto and B. Dillon, "Failure Analysis and Life Assessment of Coating: The use of mixed mode stress intensity factors in coating and 
other surface engineering life assessment," Journal of Failure Analysis and Prevention, in press DOI 10.1007/s11668-011-9525-1 (2011).

[17] K. T. Wan and Y. W. Mai, "Fracture mechanics of a new blister test with stable crack growth," Actametall urgicaet materialia, vol. 43, no. 11, pp. 4109-4115, 1995.

[18] H. M. Jahnsen, "Delamination of Coatings," (Ed. J. Lemaitre) Handbook of Materials Behavior Models, Academic Press, pp. $582-586,2001$

[19] A. A. Volinsky, et al., "Interfacial toughness measurements for thin films on substrates," Acta Materialia, vol. 50, pp. 441-466, 2002.

[20] Y. Prawoto, "Application of Linear Elastic Fracture Mechanics in Materials Science and Engineering," Lulu enterprise, North Carolina USA (2011).

[21] Y. Prawoto, N. Kamsah, M. A. Mat Yajid, and Z. Ahmad, "Energy Density Mechanics Applied to Coating Blistering Problems," Theoretical and Applied Fracture Mechanics, vol. 56, pp. 89-94, 2011

[22] J. R. Rice, "Some remarks on elastic crack-tip stress field," International Journal Solids Structure, vol. 8, pp. 751-758, 1972.

[23] H. F. Bueckner, "A novel principle for the computation of stress intensity factors," Zeitschrift fur AngewandteMathematik und Mechanik, vol. 50, pp. 529-545, 1970.

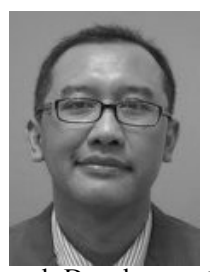

Yunan Prawoto (CEng. MImechE) was born in 1966. His last education was at the University of Missouri, USA, where he obtained his $\mathrm{PhD}$ in Mechanical Engineering in 1999. He finished his BS and MS Degrees in Keio University Japan, also at the department of mechanical engineering. He is currently with the Universiti Teknologi Malaysia. Prior to joining the UTM, he worked as a Research and Development manager at NHK Spring, USA, and also at the University of Tokyo Japan. He published more than 35-refereed scientific publications with Elsevier, Springer, Wiley, etc. He also published 2 books on Fracture Mechanics and Chief editors for several chapter books. Currently, prof. Prawoto is a registered professional engineer with the UK Council through his membership of ImechE, UK. $\mathrm{He}$ is also currently a member of IAENG. Previously he was also a registered professional engineer in the states of Missouri and Michigan, USA.

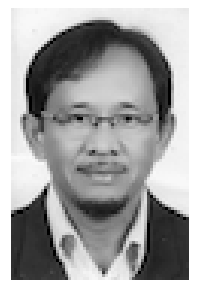

Nazri Kamsah is a member of ASHRAE. He received his Bachelor's Degree in Mechanical Engineering from University of Sunderland, United Kingdom in 1983, Masters of Engineering (Mechanical) from Universiti Teknologi Malaysia in 1988, and Ph.D in Mechanical Engineering from University of New Hampshire, Durham, USA in 2001 . He is currently a senior lecturer in the Faculty of Mechanical Engineering, Universiti Teknologi Malaysia. Prof. Kamsah areas of interest include computational solid mechanics, finite element modeling and simulation, thermal management in microelectronics, thermal comfort and energy efficiency in buildings, natural ventilation as passive cooling strategy for buildings, indoor air quality (IAQ) and computationalfluid dynamics (CFD) modeling and simulation.

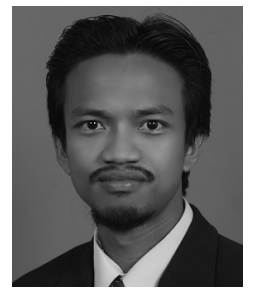

(Dr) Zaini Ahmad is currently a senior lecturer in Universiti Teknologi Malaysia, Malaysia and also a member of IAENG. He received his Master of Science (Structures, Crashworthiness \& Impact) from Cranfield University, United Kingdom in 2004. He had also completed his $\mathrm{PhD}$ in Structural Impact at Queensland University of Technology, Australia. Computational solid mechanics is of his primary research interest. Other areas of expertise are finite element modeling/simulation, crashworthiness, impact mechanics, thin-walled structures and failure of structure under dynamic loading. 